\title{
VARIATION AND PREDICTION OF LANDSCAPE PATTERN AND HABITAT CONSERVATION BASED ON CA-MARKOV AND INVEST MODEL IN HAIHE SOURCE REGION, CHINA
}

\author{
WANG, J. ${ }^{1}-\mathrm{WU}, \mathrm{T}^{1}-\mathrm{LI}, \mathrm{Q}^{2}{ }^{2}-\mathrm{WANG}, \mathrm{S} .{ }^{1 *}$ \\ ${ }^{I}$ School of Geographical Science, Shanxi Normal University, Taiyuan 030000, Shanxi Province, \\ P. R. China \\ ${ }^{2}$ Institute of Geographical Sciences, Hebei Academy of Sciences, Hebei Engineering Research \\ Center for Geographic Information Application, Shijiazhuang 050011, Heibei Province, P. R.
}

China

\author{
*Corresponding author \\ e-mail: wangsheng@sxnu.edu.cn
}

(Received 21 $1^{\text {st }}$ Jun 2021; accepted $3^{\text {rd }}$ Sep 2021)

\begin{abstract}
The fragmentation of landscape caused by urbanization and over-exploitation of water resource could result in the degradation of ecosystem services in arid and semiarid regions. Dynamic monitoring of habitat variation is of great significance to maintain regional ecological security. Habitat quality decreased from 1990 to 2015, and the mean habitat quality decreased by $1.83 \%$ in the Zhanghe River Basin in China. The habitat degradation increased during the 1990-2010 period, then decreased in 20102015. The spatial pattern of habitat quality and land use type correlated: The highest values were mainly distributed in the northern hilly region with high vegetation coverage, while the lowest values were mainly located in the southern crop and construction lands. The highest habitat degradation level occurred mainly along the roads and the agricultural areas of the peri-urban fringe. The correlation analysis indicated that slope was the dominant factor $(r=0.72, p<0.01)$ on habitat quality, followed by land use intensity $(r=-0.57, p<0.01)$. Furthermore, habitat quality was also affected by landscape pattern, and presented positive correlation with the Contagion Index, Shannon Diversity Index, Shannon Evenness Index, Landscape Shape Index and Mean Patch Size. These results could provide scientific basis for optimizing regional ecological environment and landscape management in arid and semiarid regions.

Keywords: habitat variation, model simulation, correlation analysis, landscape pattern, Zhanghe River Basin
\end{abstract}

\section{Introduction}

River areas, which provide many resources, such as fresh water, hydropower, fertile land, and support $10 \%$ of all known species (Carrizo et al., 2013), are the most productive ecosystems around the world. Unfortunately, $48 \%$ of river is facing moderate and severe fragmentation by increasingly intensive anthropogenic activities (e.g. dam construction) on a global basis (Grill et al., 2015), and causes drastic change of land use types in the basin, which could lead to biodiversity loss, habitat degradation, and produce important effect on watershed ecosystem services (Nelson et al., 2010; Seto et al., 2012). During the last decades, China has suffered the largest extinction of species, the watershed biodiversity in China continued to decline despite government efforts to alleviate threats, many precious species and habitat have lost (Liu et al., 2003). Thus, promoting the balance between habitat conservation and meeting development demands has become an increasingly urgent issue (He et al., 2017).

Habitat quality is used as an important indicator for biodiversity (Tallis et al., 2010), to some extent it can assess ecological environment change. Currently, 
researchers have effectively evaluated the variation of habitat quality and degradation at different scales, such as regional (Upadhaya et al., 2019), local (Sallustio et al., 2017), coastal zones (Zhang et al., 2020) and islands (Moreira et al., 2018). Traditional field surveys, ecological indicators and ecological model are three major methods to assess habitat quality change (Sun et al., 2019). Among these, the Integrated Valuation of Ecosystem Services and Tradeoffs (InVEST)-habitat quality (HQ) is widely used to calculate habitat quality and degradation based on land use and provides a visual tool which quantitatively evaluates biodiversity conservation, especially in areas with scarce data (Sharp et al., 2016). Sun et al. (2019) utilized InVEST model to monitor and analyze the dynamics and potential factors of habitat quality in the Nansihu Lake Basin from 1980 to 2015. Li et al. (2020) assessed the effects of urban expansion on habitat quality in the middle reaches of Heihe River from 2015 to 2030 by using the InVEST model and the slope, land use, exclusion, urban extent, transportation and hillshade (SLEUTH) model.

Land use change is the most important driver on habitat degradation (Upadhaya et al., 2019), in particular, agriculture expansion strongly affects habitat quality (FAO, 2016; Lambin et al., 2003), and this trend particularly amplifies in northern China. Previous studies have found that the newly reclaimed land and construction land in northern China kept increasing in recent 30 years (Zhao et al., 2014; Angel et al., 2011), which led to a huge loss of biodiversity. Thus, understanding the effect of land use change on habitat conservation and biodiversity is important for ecological conservation and management (Sun et al., 2019). The CA-Markov model is the most widely applied tool for simulation and predication of land use patterns, and it integrates the complex spatial systems analysis capabilities of the CA model with the long-term forecasting techniques of the Markov model (Batty and Xie., 1994; Luijten., 2003). A number of studies have effectively applied CA-Markov to simulate land use in different spatial and temporal scale, such as, Nourqolipour et al. (2015) used CA, multi-criteria evaluation (MCE), and Markov chain (MC) to simulate the expansion of oil palm in the Kuala Langat district, Malaysia. Chen et al. (2018) simulates the future landscape pattern in Hubei Section of Three Gorges Reservoir area in 2020. Cheng et al. (2020) utilized the CA-Markov model to simulate the evolution trend of the land to propose the ecological division of the main urban area of Jinzhong in Shanxi Province.

In this paper, the spatio-temporal change of habitat quality and degradation was analyzed in Zhanghe River Basin, which was an important water conservation source for local and downstream areas. Zhanghe River Basin has experienced rapid urban expansion and over-exploitation of mineral resources, facing severe water resource shortage and prominent water conflicts, which has resulted in serious ecological threats. Previous studies focused on assessing the change of water quality, runoff, and sediment under the climate change and land use change. However, few studies were concerned with dynamic monitoring habitat conservation change. Thus, this study aimed to (1) assess habitat quality and degradation from 1990 to 2030 in the Zhanghe River Basin; (2) explore the main impact factors on habitat quality; and (3) analyze the role of the landscape pattern indexes on affecting habitat quality. The research fills a knowledge gap about the impact of anthropogenic activities (especially landscape pattern) on biodiversity status by adopting an integrative approach. This paper is helpful to understand the importance of landscape structure and pattern on habitat quality, and guide habitat conservation and management in arid/semi-arid areas facing serious water conflicts. 


$$
-4585 \text { - }
$$

\section{Materials and methods}

\section{The Zhanghe River Basin}

Zhanghe River $\left(112^{\circ} \sim 114^{\circ} 30^{\prime} \mathrm{E}, 35^{\circ} 30^{\prime} \sim 38^{\circ} \mathrm{N}\right)$ is an important water conservation source for local and downstream areas, which are located in the southwest of Haihe River Basin, China (Fig. 1). It spans a total length of $460 \mathrm{~km}$ and has a drainage area of about $17874 \mathrm{~km}^{2}$. Cropland, forestland, grassland, construction land and water bodies occupy $40 \%, 23 \%, 33 \%, 3 \%$, and $0.9 \%$ in 2015, respectively (Wang et al., 2021). The basin is species-rich, including 40 families and 117 genera of bird species, and 26 of them are classified in the first or second levels of national protected birds, and is the home for several threatened wildlife species, such as the leopard and the taiga musk deer. Additionally, there are two or three hundred years of natural forest communities, and some wild plants, such as the Juglans mandshurica, Amur linden and Acanthopanax senticosus. The basin is rich in mineral resources, including coal, iron, aluminum, and other mineral resources. In recent years, water resource and biodiversity face severe threats under the impact of intensive human activities.

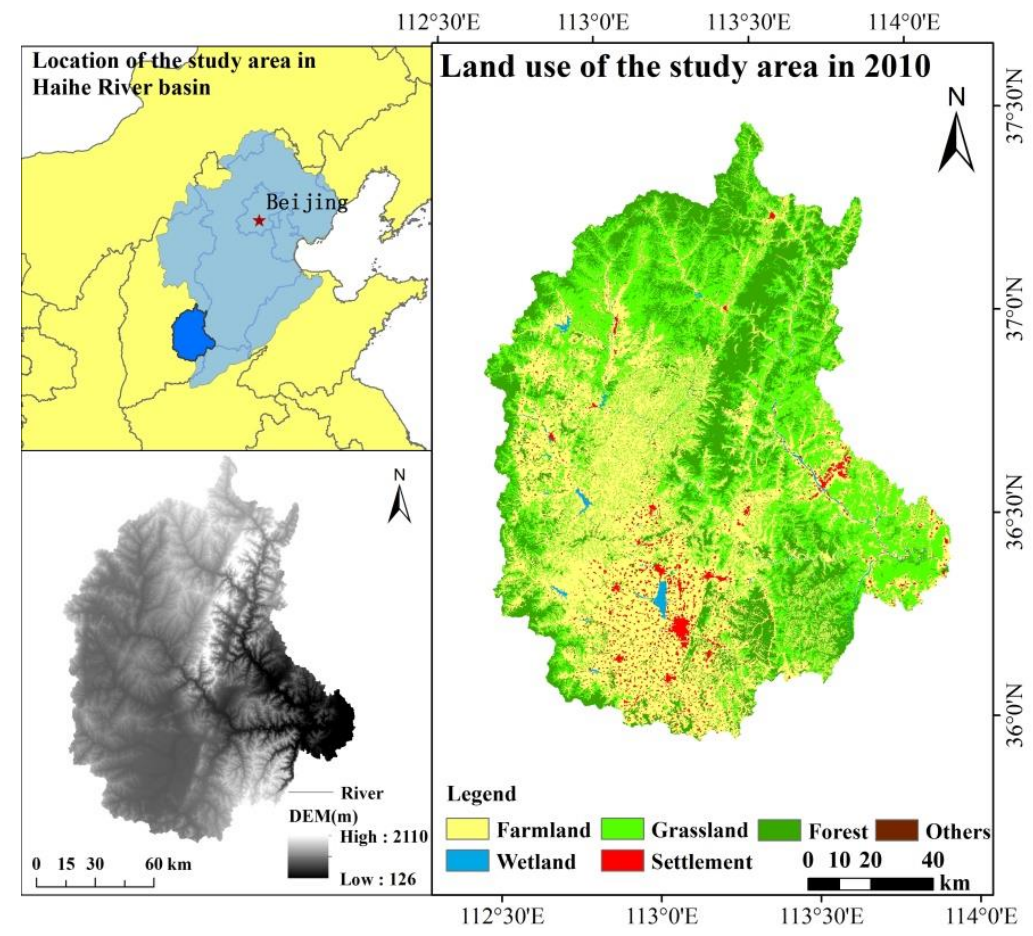

Figure 1. Geographic location of Zhanghe River Basin of China

\section{Data collection}

Land use data in 1990, 1995, 2000, 2005, 2010 and 2015 of Zhanghe River Basin were obtained from the Resource and Environmental Data Cloud Platform (http://www.resdc.cn/), with a spatial resolution of $1 \mathrm{~km}$. The digital elevation model (DEM) data with $90 \mathrm{~m}$ resolution was downloaded from the Geospatial Data Cloud. A normalized difference vegetation index map (NDVI), a map of gross domestic product (GDP), and a population distribution map were collected by the Data Center for Resources and Environmental Sciences from the Chinese Academy of Sciences 


$$
-4586-
$$

(RESDC) (http://www.resdc.cn). Main roads and railways were derived from the national fundamental geographic information system (http://nfgis.nsdi.gov.cn/).

\section{Landscape pattern analysis}

The effects of landscape patterns on ecological security are increasingly recognized. In this study, the landscape pattern indexes were used to assess the effect of LUCC on habitat quality in the Zhanghe River Basin by using FRAGSTATS version 4.2.1. Based on the relevant study (Han et al., 2019; Huang et al., 2020) and the scale of the study area, the variation of landscape pattern in Zhanghe River Basin was explored from two levels, including the class level and landscape level, and 8 indices were selected to describe landscape structure and patterns. At the class level, we chose the Number of Patches (NP), Patch Density (PD), Mean Patch Size (MPS), and Landscape Shape Index (LSI). At the landscape level, the Contagion Index (CONTAG), Shannon Diversity Index (SHDI), Shannon Evenness Index (SHEI), and Edge Density (ED) were calculated.

\section{The InVEST-habitat quality module}

InVEST-HQ module was used to assess the habitat quality and degradation degree of Zhanghe River Basin (Sharp et al., 2016). This model was developed at Stanford University, The Nature Conservancy and World-Wildlife Fund (Zhang et al., 2020), and was used to detect the impact of human activities on ecosystem based on suitability and threats and assumed that areas with high habitat quality would support more species (Terrado et al., 2016). The calculation method is as follows:

$$
\begin{aligned}
D_{x j}=\sum_{1}^{r} \sum_{1}^{y}\left(\frac{\omega_{r}}{\sum_{r=1}^{n} \omega_{r}}\right) \times r_{y} \times i_{r x y} \times \beta_{x} \times S_{j r} \\
i_{r x y}=\left\{\begin{array}{l}
1-\left(\frac{d_{x y}}{d_{r \max }}\right) \\
\exp \left[-\left(\frac{2.99}{d_{r \max }}\right) \times d_{x y}\right]
\end{array}\right.
\end{aligned}
$$

where $\mathrm{r}$ is the number of threats $(\mathrm{r}=1,2,3 \ldots \mathrm{R}) ; \omega_{r}$ is the impact weight of threat $r ; r_{y}$ is the number of threat factors on each grid of land classification layers; $i_{r x y}$ is the degradation decay function through distance, which can be expressed as the linear or exponential function of distance from threats to habitats; $\beta_{x}$ is the accessibility level of pixel $x ; \mathrm{s}_{j r}$ represents the sensitivity of habitat type $j$ to threat $r$. $\mathrm{d}_{x y}$ is the linear distance between $x$ and $y ; \mathrm{d}_{r m a x}$ is the maximum effective distance of threat $r$.

$$
Q_{x j}=H_{j}\left[1-\left(\frac{D_{x j}^{z}}{D_{x j}^{z}+k_{z}}\right)\right]
$$

where $\mathrm{Q}_{x j}$ is the habitat quality of raster $x$ in land use type $j$; $\mathrm{H}_{j}$ is the habitat suitability of land use type $j, \mathrm{D}_{x j}^{z}$ is the threat level of raster $x$ in land use type $j ; K$ and $Z$ is the 
half-saturation constant and the scaling parameter to reflect the spatial heterogeneity, respectively.

The main data of the InVEST-HQ module include current LUCC, threats factor layers, index of threats and sensitivity, weight of threat factor, distance from threat (Zhang et al., 2020). For this study, we assumed that forest, grassland, and water provided better habitat conservation than agriculture and construction land (Upadhaya et al., 2019), so we set agriculture, construction land as the major threat factors. In addition, we also selected main roads and railways as threat factors (Table 1). The index of threats, sensitivity and other parameters were derived from cognate studies (Chen et al., 2020; Wu et al., 2015; Wang et al., 2019) (Table 2).

Table 1. Maximum distance and weight of the threats affecting habitat quality

\begin{tabular}{c|c|c|c}
\hline Threat factor & Maximum effective distance & Weight & Decay \\
\hline Agriculture & 2 & 0.6 & Linear \\
Construction land & 8 & 1 & Exponential \\
Main road & 3 & 0.6 & Exponential \\
Main railway & 5 & 0.6 & Exponential \\
\hline
\end{tabular}

Table 2. The sensitivity of landscape types to each threat factor

\begin{tabular}{c|c|c|c|c|c}
\hline Land use type & Habitat & Agriculture & Construction land & Main road & Main railway \\
\hline Agriculture & 0.4 & 0.3 & 0.5 & 0.7 & 0.6 \\
Forestland & 1 & 0.6 & 0.8 & 0.7 & 0.8 \\
Grassland & 0.85 & 0.4 & 0.7 & 0.5 & 0.4 \\
Water body & 1 & 0.4 & 0.8 & 0.6 & 0.5 \\
Construction land & 0 & 0 & 0 & 0 & 0 \\
\hline
\end{tabular}

\section{The CA-Markov model}

The CA-Markov model is an effective tool to predict LUCC. It considers spatial distribution of each land type and geographic directions (Mansour et al., 2020). We applied the CA-Markov module of IDRISI 17 to predict LUCC in 2030. To obtain the projected LUCC in 2030, we prepared suitability map for any land use type using MultiCriteria-Evaluation (MCE). Under the background of fast urbanization and land use policy, it was assumed that current construction land and water remained unchanged, while the multiple ecological land was transformed into urban land. We have defined transition rules for any land use types considering a number of factors (e.g. elevation, slope, distance to road and railway, distance to city, population, and GDP).

In this paper, we set LUCC in 2000 as a starting year with the transition probability matrix of 1990-2000 and the suitability maps of each land use type to predict LUCC in 2010. The Kappa index was used to validate the accuracy of the projected LUCC in 2010 with the observed LUCC in 2010. The result (Table 3) showed the simulation generally matched well for each class type, so, the model could predict future land use in a reliable way. Therefore, based on the reliable simulation of LUCC in 2010, we used the transition probability matrix of 2000-2010 and the 2010 land use map to predict LUCC in 2030. 
Table 3. Observed and simulated LUCC of Zhanghe River Basin in 2010

\begin{tabular}{c|c|c|c|c|c}
\hline Land use type & Agriculture & Forestland & Grassland & Water body & Construction land \\
\hline Actual area & 7213.07 & 4168.26 & 5829.49 & 160.43 & 497.37 \\
Simulate area & 7207.21 & 4047.70 & 5744.05 & 129.58 & 502.29 \\
Kappa & 0.96 & 0.92 & 0.99 & 0.80 & 0.75 \\
\hline
\end{tabular}

\section{Results}

\section{Landscape pattern indexes change}

Extensive human activities in the Zhanghe River Basin have resulted in profound change in the size, number, and spatial distribution of landscape patterns, with the increase in habitat degradation (Han et al., 2019). Based on the statistical results (Table 4), NP, PD and ED increased by $3.58 \%, 2.75 \%$ and $1.38 \%$ from 1990 to 2010 , respectively, then fell in 2015, which indicated that the watershed faced severe landscape fragmentation and heterogeneity during the 1990-2010 period, while slowed down in recent years. SHDI and SHEI showed an increasing tendency, and increased by 0.0891 and 0.0498 , respectively. By contrast, CONTAG decreased continuously from 49.3957 to 46.5833 , which reflected increased landscape diversity and heterogeneity in the last 25 years.

Table 4. Landscape pattern indices of landscape level

\begin{tabular}{c|c|c|c|c|c|c}
\hline Year & NP & PD & ED & CONTAG & SHDI & SHEI \\
\hline 1990 & 16555 & 0.9295 & 36.4358 & 49.3957 & 1.1975 & 0.6683 \\
2000 & 16584 & 0.9398 & 36.5753 & 49.2784 & 1.1999 & 0.6697 \\
2005 & 16947 & 0.9516 & 36.8563 & 48.7547 & 1.2133 & 0.6772 \\
2010 & 17114 & 0.9628 & 36.9402 & 48.6638 & 1.2153 & 0.6783 \\
2015 & 17108 & 0.9567 & 36.5693 & 46.5833 & 1.2866 & 0.7181 \\
\hline
\end{tabular}

At the class level (Fig. 2), the landscape with the highest NP, PD and LSI was grassland, the maximum appeared in 2010 and then decreased from 2010 to 2015, while MPS increased by $3.51 \%$, demonstrating that landscape of grassland spatially centralized due to the GGP. NP and PD of agricultural land increased by $5.53 \%$ and $5.08 \%$, while MPS decreased by $8.16 \%$, which indicated that the degree of agriculture patches fragmentation increased in the past 25 years. NP and PD of forest land showed no obvious change, while MPS decreased, mainly manifested that forest land experienced fragmentation to a certain extent. NP and PD of construction land decreased, and MPS increased, which showed that construction land tended to expand and concentrate. Further analysis of the patch change based on LSI showed that construction land experienced large change. From 1990 to 2000, LSI of construction land showed no obvious change, while increased during 2005-2010 and then decreased in 2015.

\section{Spatial distribution of habitat quality and degradation}

The habitat quality value was between 0 and 1 , and the closer to 1 , the higher the habitat quality. Overall, the level of habitat quality was comparatively high since forestland and grassland accounted for about 58\%, while decreased from 1990 to 2015 in 
the Zhanghe River Basin (Fig. 3a). The value of habitat quality showed slight decreasing trend during 1990-2010, with a rate of $0.15 \%$, and then fell dramatically. The mean habitat quality decreased by $1.83 \%$ during $1990-2015$. From the perspective of the spatial distribution pattern, the distribution of habitat quality was correlated with land use type. Specifically, the lowest habitat quality was mainly consistent with construction land and agricultural land, which was mainly distributed in the central and western plain, while the highest habitat quality was distributed in the northern and southeast mountain which were thickly forested and grassed (Fig. 4). Furthermore, based on the natural breaks method and cognate studies (He et al., 2017; Tang et al., 2020), we divided habitat quality into five classes: low quality (0-0.25), relatively low (0.26-0.65), medium (0.66-0.85), relatively high (0.86-0.95) and high quality (0.96-1.0). In 1990, the area with low and relatively low habitat quality accounted for $43.4 \%$, while the high and relatively high accounted for only $24.2 \%$ of all habitats. In 2015, the area with low and relatively low habitat quality increased by $1 \%$, while the high and relatively high habitat quality area decreased by $0.7 \%$, and the medium grade decreased by $0.58 \%$. This indicated that the habitat quality declined mainly due to the contraction of high and medium grades.

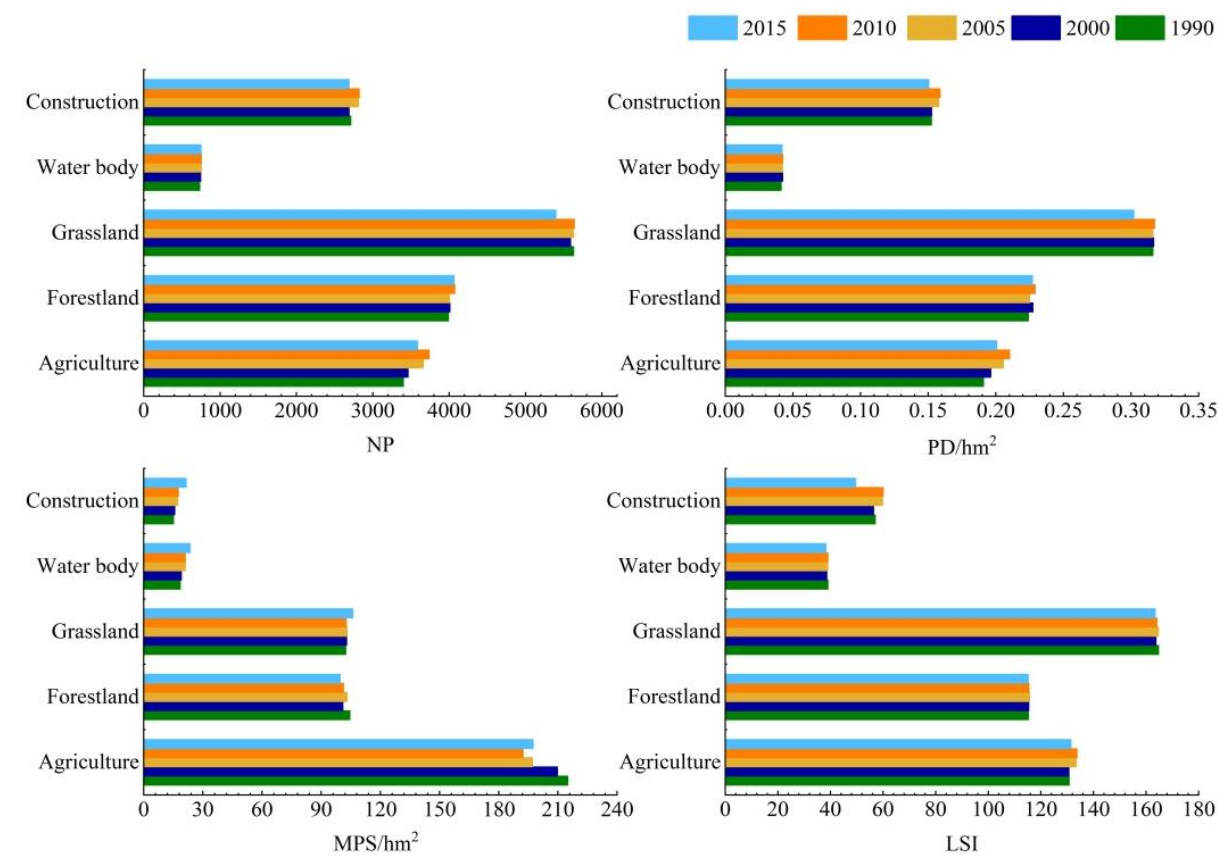

Figure 2. Landscape indices of class metrics level

The habitat degradation increased from 1990 to 2015, with the highest habitat degradation of 0.23 in the Zhanghe River Basin (Figs. $3 b$ and 5). The trend of habitat degradation increased in 1990-2010 and then decreased in 2010-2015, and the value of habitat degradation increased by $0.37 \%$ from 1990 to 2015 . The main reason is that the agricultural land converted into grassland owing to the GGP, which alleviated habitat degradation. But the habitat quality of grassland was relatively low compared to forestland, and the habitat degradation increased during 1990-2015 in the Zhanghe River Basin. The spatial patterns manifested that the high and relatively high grades of habitat degradation were mainly concentrated in the roads and farmland of urban fringe, indicating the negative impact of anthropogenic activities on the ecosystem. The low 
grade was mainly located in the north and southeast owing to the mountainous terrain and implementation of the GGP. The median grade was mainly distributed in the central region, where the landscape types were dominated by agriculture. Based on the natural breaks method (He et al., 2017; Tang et al., 2020), the habitat degradation in Zhanghe River Basin was divided into five levels: low quality (0-0.02), relatively low (0.03-0.04), medium (0.05-0.08), relatively high (0.09-0.10) and high quality (0.11-0.23). From 1990 to 2015 , the low grade increased by $37.8 \mathrm{~km}^{2}$, the medium grade decreased by 198.67 $\mathrm{km}^{2}$, while the high and relatively high grade increased by $169.49 \mathrm{~km}^{2}$, which indicated that most medium grade converted to high and relatively high grade and caused the degradation of watershed habitat conservation. In other words, a number of agricultural lands replaced by urban land caused the watershed ecological environment deterioration.
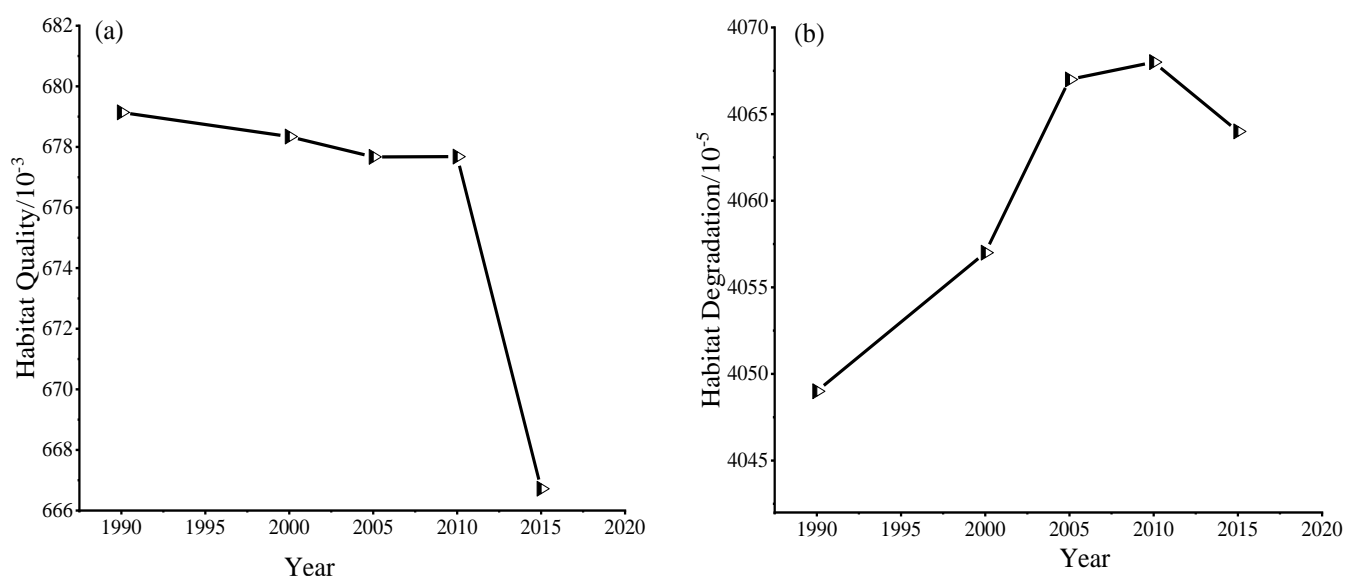

Figure 3. The mean habitat quality and degradation from 1990 to 2015 in the Zhanghe River Basin
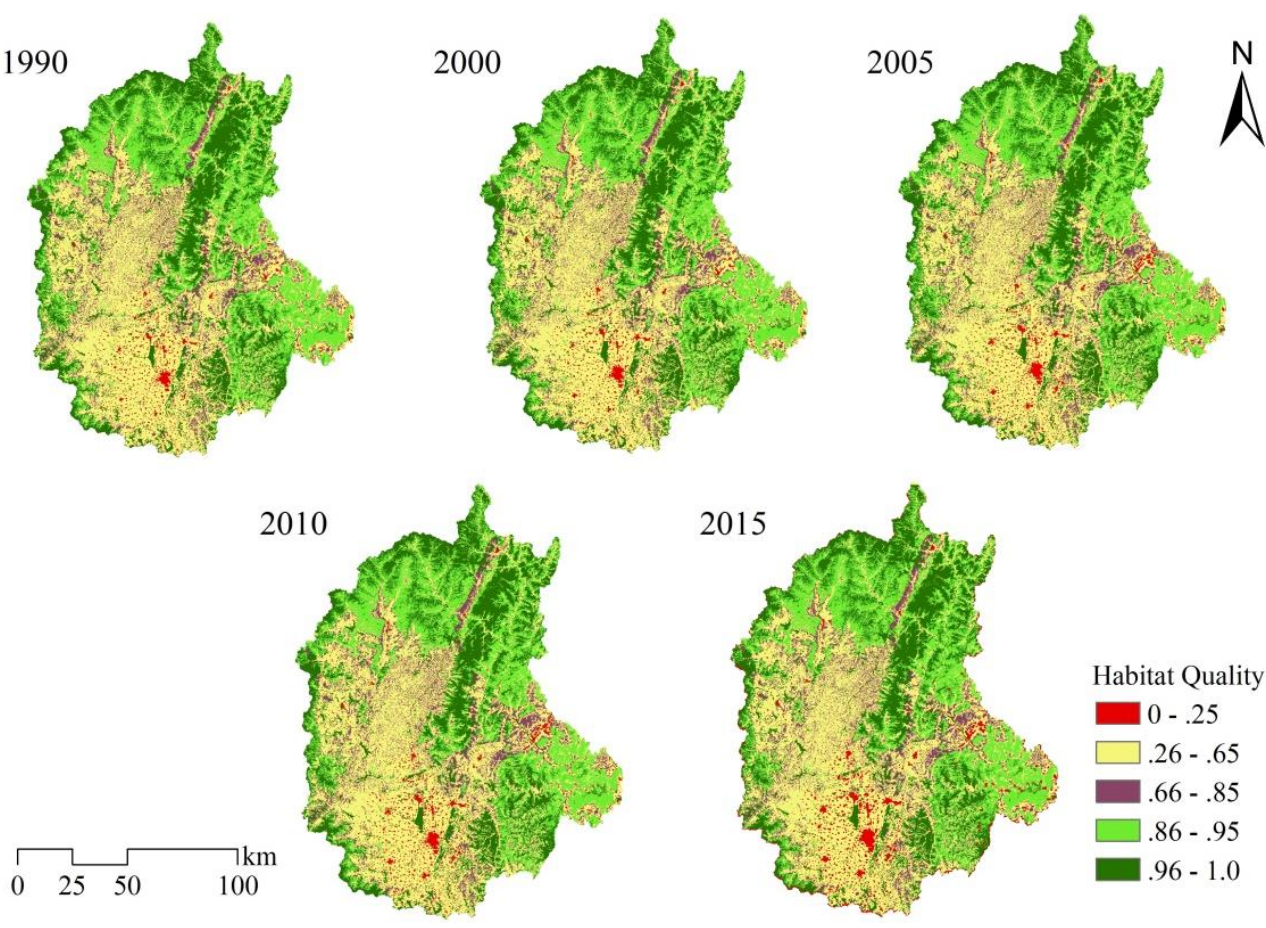

Figure 4. The distribution pattern of habitat quality from 1990 to 2015 


$$
-4591-
$$
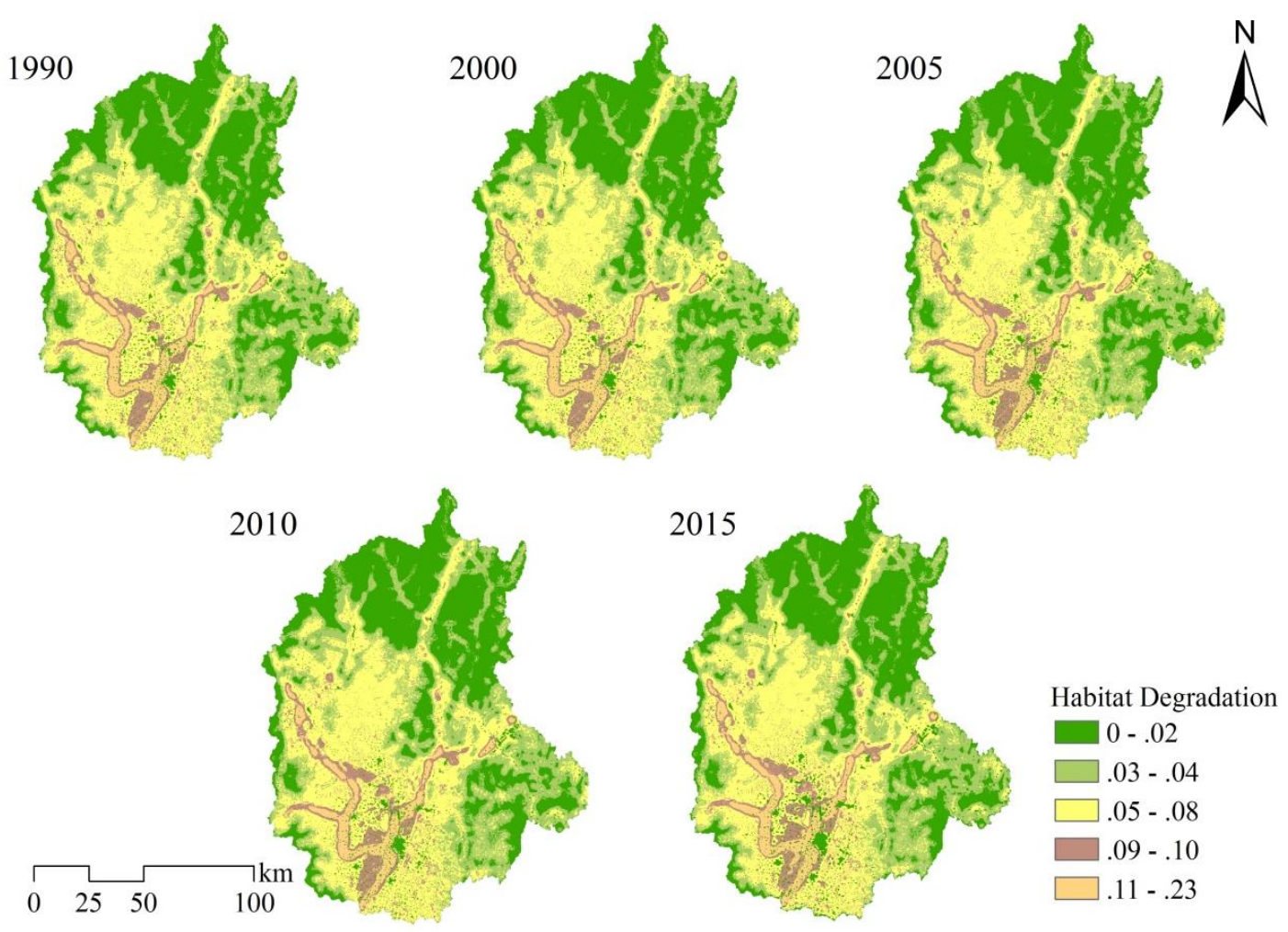

Figure 5. The distribution pattern of habitat degradation from 1990 to 2015

From the perspective of the spatial pattern, the decrease of habitat quality showed obvious spatial aggregation, and mainly concentrated in the peri-urban fringe and agricultural land (Figs. 6 and 7). Further analysis of the spatial change process was carried out based on Moran's $I$ and LISA statistics, which showed that habitat loss in the Zhangehe River Basin from 1990 to 2015 mainly owed to the decline of agricultural land. The Moran's $I$ of habitat quality decreased from 0.202 in 1990 to 0.191 in 2015, indicating that the spatial aggregation of habitat quality was poor and tended to be dispersed. The Moran's I of habitat degradation was more than 0.8 during 1990-2015, demonstrating that the spatial aggregation was significant, but there was a tendency of dispersion (the value decreased from 0.867 in 1990 to 0.840 in 2015). LISA statistics was used to identify local cluster. The high-high aggregation area of habitat quality was concentrated in the central forestland with mountain terrain and less human activities in 2015, while the low-low area was mainly located in the southwest with agricultural land. And most of the high-high aggregation area of habitat degradation was distributed in agricultural land, indicating that agricultural activities posed a great threat to the habitat conservation in Zhanghe river basin.

\section{Forecast of landscape pattern and habitat status}

Figure 8 showed the spatial distribution of LUCC, habitat quality and degradation in 2030. Based on land use policy and urban expansion, the agricultural land and construction land increased by $134.28 \mathrm{~km}^{2}$ and $226.5 \mathrm{~km}^{2}$, while forestland and grassland decreased by $160.42 \mathrm{~km}^{2}$ and $531.91 \mathrm{~km}^{2}$, respectively. Construction land was mainly converted from agricultural land, while forestland and grassland converted to agricultural land. 
(a)

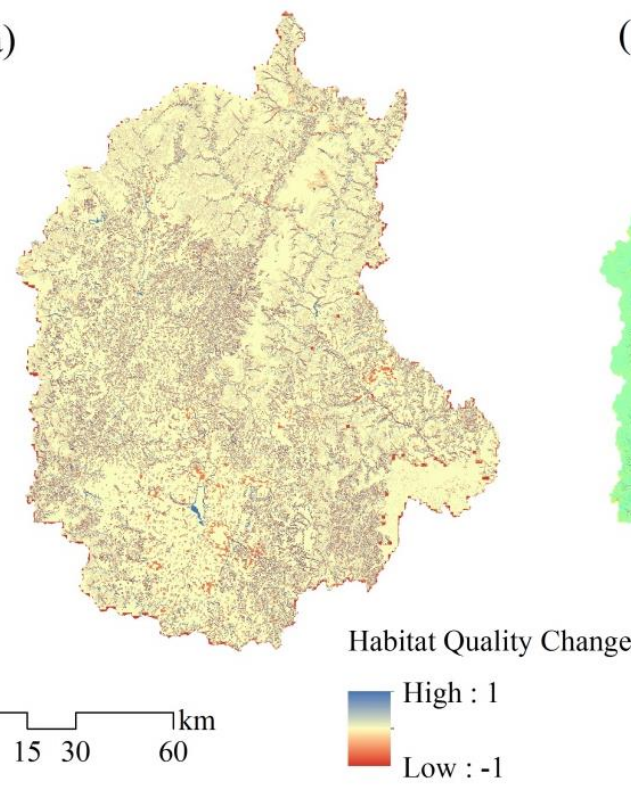

(b)

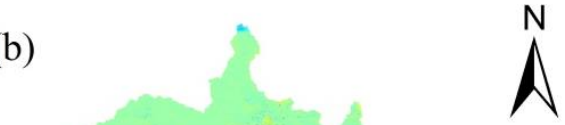

$\bigwedge^{N}$

Figure 6. The spatial change pattern for habitat quality and degradation in the Zhanghe River

Basin
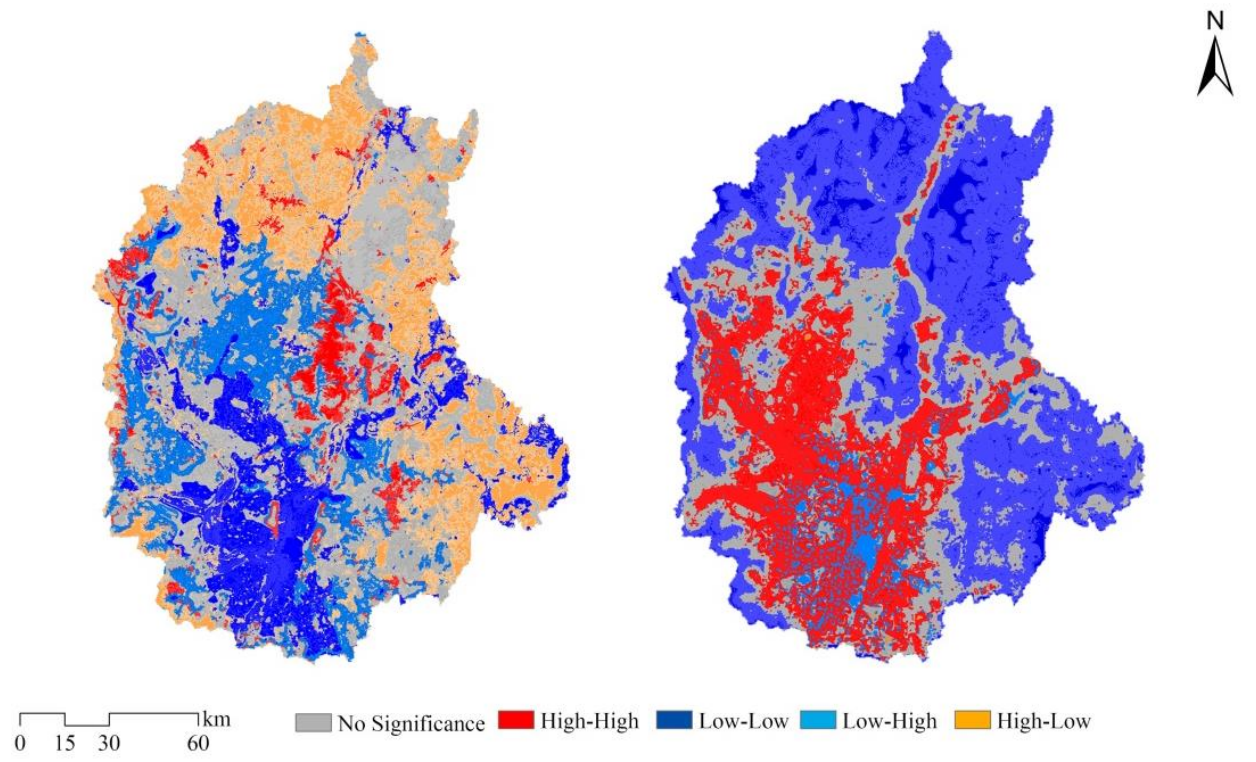

Habitat Degradation Change

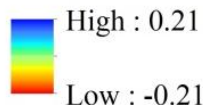

Figure 7. LISA aggregation in 2015: (left) habitat quality, (right) habitat degradation

The overall ecological environment continued to decline in 2030, and the mean value of habitat quality and degradation was 0.6535 and 0.0423 , respectively (Table 5). Compared to 2015, the area with low and relatively low habitat quality increased by $342.62 \mathrm{~km}^{2}$, but the area with medium value decreased by $410.23 \mathrm{~km}^{2}$, and the area with high and relatively high habitat degradation increased by $314.42 \mathrm{~km}^{2}$. The results indicated that the decline of ecological environment was mainly due to the conversion of medium grade to low grade. Thus, under current land use policy, we should do more effort to balance economic development and habitat conservation. 


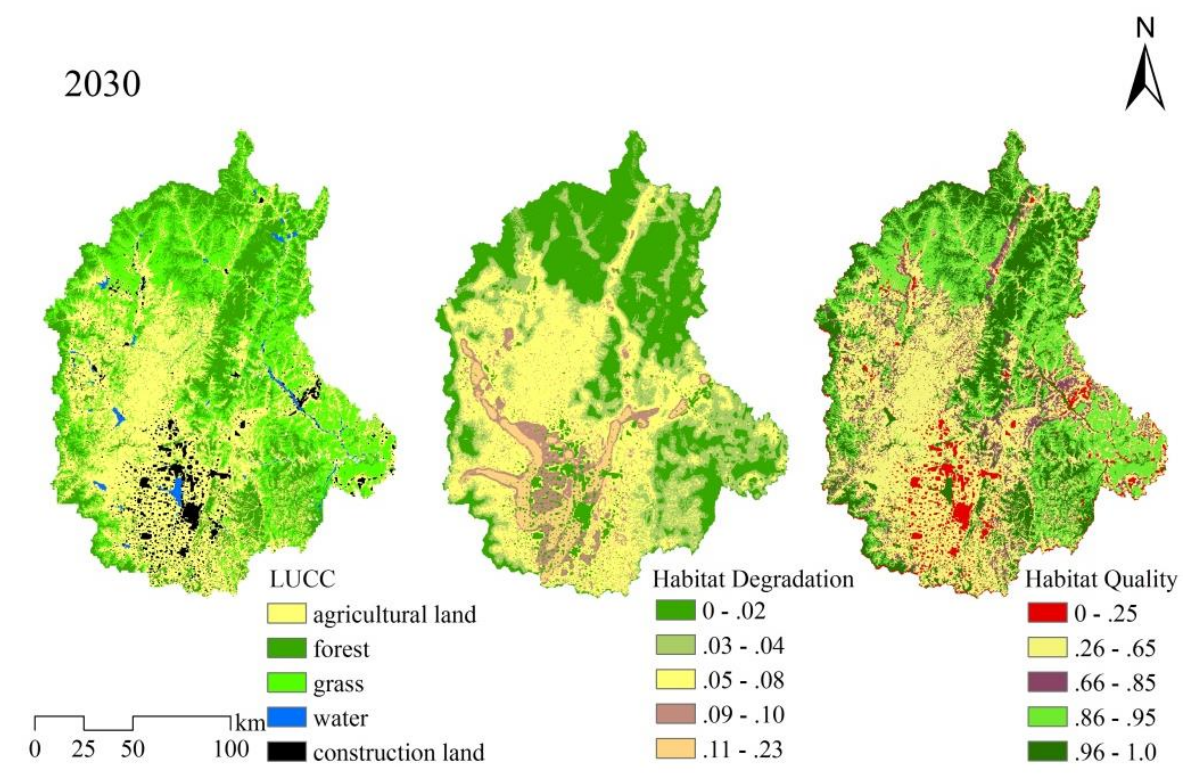

Figure 8. Land use map, habitat quality and degradation for 2030

Table 5. Area of the landscape under different habitat quality and degradation classes in $2030\left(\mathrm{~km}^{2}\right)$

\begin{tabular}{c|c|c|c|c|c}
\hline & Low & Relatively low & Medium & Relatively high & High \\
\hline Habitat quality & 1051.89 & 7227.28 & 5347.78 & 40.1 & 4195.31 \\
Habitat degradation & 6940.69 & 3320.05 & 5702.95 & 1014.39 & 911.75 \\
\hline
\end{tabular}

\section{Discussion}

Habitat quality could reflect regional change of biodiversity and is regarded as proxy, and the geographical factors and human activities are essential to understand the change of habitat quality and degradation (Sun et al., 2019). To investigate the potential impact factors on habitat quality, 6 typical impact factors were listed (Table 6). In this paper, we subdivided the watershed into Hydrological Response Units (HRUs) based on the SWAT model (Wang et al., 2021), and a total of 872 units were formed, then the correlations between the habitat quality and the impact factors were analyzed based on HRUs by using Pearson correlation coefficient. The results showed that habitat quality and the geographical factors (DEM, slope and NDVI) presented significant positive correlation, while habitat quality was significantly negative with human activities, such as GDP, population density and intensity of land use (Table 6). Slope was the dominant factor, followed by intensity of land use and NDVI. The flat terrain and steep slope regions were consistent with forestland and grassland regions with less human activities. Thus, habitat quality in mountains was better than in plain areas. Land use intensity (Hu et al., 2013) is an important index for the level of anthropogenic disturbance. Construction land and agricultural land distributed mainly in the plain and were greatly affected by human activities. Correspondingly, these regions presented dramatic habitat degradation. The related coefficient between habitat quality and other socio-economic factors (e.g. GDP and population density) further indicated that intensive human activities could cause habitat degradation in the basin (Sun et al., 2019). 
Table 6. The related coefficient between distribution of habitat quality and impact factors in 2015

\begin{tabular}{c|c|c|c|c|c|c}
\hline Impact factors & DEM & Slope & NDVI & GDP & Population & Intensity of land use \\
\hline Related coefficient & $0.53^{* *}$ & $0.72^{* *}$ & $0.55^{* *}$ & $-0.45^{* *}$ & $-0.33^{* *}$ & $-0.57^{* *}$ \\
\hline
\end{tabular}

**Correlation is significant at the 0.01 level

The landscape composition and configuration played an important role on exploring regional habitat quality variation. In this study, we further analyzed the correlation between landscape patterns and habitat quality (Table 7). Habitat quality presented a significant positive correlation with CONTAG, SHDI and SHEI, which indicated that habitat quality would increase with better landscape diversity, evenness, and contagion. The correlation between habitat quality and LSI, MPS was slight but obvious, which reflected that larger mean patch size, better habitat quality, and complexity of landscape shapes increased the heterogeneity of habitat quality. Agricultural land was the main land use type in the Zhanghe River Basin, but the increasing fragmentation of agricultural land caused the decline of ecological system function and habitat degradation. In addition, along with deforestation and urban expansion, the large patches of forestland became smaller, while the patches of construction land tended to expand, which led to the decline of habitat quality.

Table 7. The correlation coefficient between distribution of habitat quality and impact factors

\begin{tabular}{c|c|c|c|c|c|c}
\hline \multicolumn{7}{c}{ At the landscape level } \\
\hline Landscape metrics & NP & PD & ED & CONTAG & SHDI & SHEI \\
Related coefficient & -0.57 & -0.44 & 0.19 & $0.98^{* *}$ & $0.99^{* *}$ & $0.99^{* *}$ \\
\hline \multicolumn{7}{|c|}{ At the class level } \\
\hline Landscape metrics & NP & PD & LSI & MPS & & \\
Related coefficient & -0.01 & -0.07 & $0.14^{* *}$ & $0.11^{* *}$ & & \\
\hline
\end{tabular}

**Correlation is significant at the 0.01 level

The Zhanghe River Basin has undergone habitat degradation and fragmentation during 1990-2015. Based on current land use policy and the urban expansion trend, LUCC would continue to fragment, and the habitat degradation would become more severe. Therefore, the balance between habitat conservation and urbanization was the key to socio-economic sustainable development. More effective habitat conservation policies and integrated landscape management strategies should be adopted in the future.

\section{Conclusion}

Habitat quality and degradation are important indicators for exploring watershed ecological status. In this study, Zhanghe River Basin, with severe water conflicts, was selected to investigate characteristics, prediction, and potential causes of habitat variation by using CA-Markov and the InVEST model. 
The results showed that Zhanghe River Basin has experienced landscape fragmentation and habitat loss during 1990-2015, and the area with habitat degradation existed in the peri-urban fringe and agricultural land. Specifically, the agriculture and forestland fragmentation increased, while grassland and construction land tended to expand and concentrate, and the mean habitat quality decreased by $1.83 \%$ from 1990 to 2015. The overall distribution pattern of habitat quality in the basin was significantly related to spatial distribution of land use types: the low grade of habitat quality was mainly consistent with construction land and agricultural land, while forestland and grassland with higher habitat quality were distributed in the northern mountainous area. The area with high grade habitat degradation was mainly existed in the roads and the agriculture in peri-urban fringe. The simulated LUCC and habitat quality/degradation map for 2030 indicated that the overall habitat quality continued to decline under current land use policy and urban expansion. The potential impact factors on habitat quality were further analyzed, which showed that terrain was the main factor, intensity of land use was the secondary factor, and other factors (e.g. NDVI, GDP, Population) also had an important impact on the habitat quality. From the perspective of landscape pattern, the CONTAG, SHDI, SHEI, LSI and MPS showed more significant correlation with habitat quality. If the land use policy and urban expansion do not change, the ecological environment will continue to deteriorate.

In the following research, we will use the InVEST model to further explore the ecosystem service functions such as soil conservation, carbon fixation and food production in the Zhanghe River Basin to provide feasible suggestions for ecological environment protection in the Zhanghe River Basin.

Acknowledgments. This research was funded by the National Key Research and Development Program of China (2020YFF0305905), the National Natural Science Foundation of China (No. 41801034; 41801007), Shanxi philosophy and social science planning project (2020YY069, 2020YY070), the Science and Technology program of Linfen City (2060499). We would like to extend special thanks to the editor and reviewers for insightful advice and comments on the manuscript.

\section{REFERENCES}

[1] Angel, S., Parent, J., Civco, D. L., Blei, A., Potere, D. (2011): The dimensions of global urban expansion: estimates and projections for all countries 2000-2050. - Progress in Planning 75: 53-107.

[2] Batty, M., Xie, Y. (1994): From cells to cities. - Environment and Planning B: Planning and Design 21: 31

[3] Carrizo, S. F., Smith, K. G., Darwall, W. R. T. (2013): Progress towards a global assessment of the status of freshwater fishes (Pisces) for the IUCN Red List: application to conservation programs in zoos and aquariums. - International Zoo Yearbook 47: 4664.

[4] Chen, Y. J., Zhao, L., Tao, J. Y., Zhang, P. T. (2020): Habitat quality evaluation before and after unused land development based on the InVEST model: a case study of Tang Country. - Chinese Journal of Eco-Agriculture 28(7): 1093-1102.

[5] Cheng, C. N., Hu, Y., Feng, Y., Zhao, M. (2020): Construction of urban ecological zones based on CA-Markov model: a case study of the main urban area of Jinzhong. - Acta Ecological Sinica 40(4): 1455-1462. 
[6] Chun, L., Sun, T., Wang, T., Li, Z., Cai, C. (2018): Evolution and prediction of landscape pattern and habitat quality based on CA-Markov and InVEST model in Hubei Section of Three Gorges Reservoir Area. - Sustainability 10: 3854.

[7] FAO (2016): Global Forest Resources Assessment 2015. - FAO Forestry Paper No. 1.

[8] Grill, G., Lehner, B., Lumsdon, A. E., MacDonald, G. K., Zarfl, C., Reidy, L. C. (2015): An index-based framework for assessing patterns and trends in river fragmentation and flow regulation by global dams at multiple scales. - Environmental Research Letters 10: 015001 .

[9] Han, Y. W., Kang, W. M., Thorne, J., Song, Y. K. (2019): Modeling the effects of landscape patterns of current forests on the habitat quality of historical remnants in a highly urbanized area. - Urban Forestry and Urban Greening 41: 354-363.

[10] He, J. H., Huang, J. L., Li, C. (2017): The evaluation for the impact of land use change on habitat quality: a joint contribution of cellular automata scenario simulation and habitat quality assessment model. - Ecological Modelling 366: 58-67.

[11] Hu, H., Liu, H., Hao, J., An, J. (2013): Spatio-temporal variation in the value of ecosystem services and its response to land use intensity in an urbanized watershed. Acta Ecologica Sinica 33 (8): 2565-2576.

[12] Huang, M. J., Yue, W. Z., Feng, S. R., Zhang, J. H. (2020): Spatial-temporal evolution of habitat quality and analysis of landscape patterns in Dabie Mountain area of west Anhui province based on InVEST model. - Acta Ecologica Sinica 40(9): 1-12.

[13] Lambin, E. F., Geist, H. J., Lepers, E. (2003): Dynamics of land-use and land-cover change in tropical regions. - Annual Review of Environment and Resources 28: 205-241.

[14] Li, S. G., Wang, L. C., Yan, C. X., Liu, H. Y. (2020): Simulation of urban spatial expansion in oasis towns based on habitat quality: a case study of the middle reaches of Heihe River. - Acta Ecologica Sinica 49(9): 1-12.

[15] Liu, J., Ouyang, Z., Pimm, S. L., Raven, P. H., Wang, X., Miao, H., Han, H. (2003): Ecology Protecting China's biodiversity. - Science (New York) 300(5623): 1240-1241.

[16] Luijten, J. C. (2003): A systematic method for generating landuse patterns using stochastic rules and basic landscape characteristics: results for a Colombian Hillside Watershed. - Agriculture Ecosystems \& Environment 95(2-3): 427-441.

[17] Mansour, S., Al-Belushi, M., Al-Awadhi, T. (2020): Monitoring land use and land over changes in the mountainous cities of Oman using and CA-Markov modelling techniques. - Land Use Policy 91: 104414.

[18] Moreira, M., Fonseca, C., Vergilio, M., Calado, H., Gil, A. (2018): Spatial assessment of habitat conservation status in a Macaronesian island based on the InVEST model: a case study of Pico Island (Azores, Portugal). - Land Use Policy 78: 637-649.

[19] Nelson, E., Sander, H., Hawthorne, P., Conte, M., Ennaanay, D., Wolny, S., Manson, S., Polasky, S. (2010): Projecting global land-use change and its effect on ecosystem service provision and biodiversity with simple models. - PLoS One 5(12): e14327.

[20] Nourqolipour, R., Shariff, A. R. B. M., Balasundram, S. K., Ahmad, N. B., Sood, A. M. (2015): A GIS-based model to analyze the spatial and temporal development of oil palm land use in Kuala Langat district, Malaysia. - Environmental Earth Sciences 73(4): 16871700.

[21] Sallustio, L., De Toni, A., Strollo, A., Di Febbraro. M., Gissi, E., Casella, L., Geneletti, D., Munafo, M., Vizzarri, M., Marchetti, M. (2017): Assessing habitat quality in relation to the spatial distribution of protected areas in Italy. - Journal of Environmental Management 201: 129-137.

[22] Sharp, R., Chaplin-kramer, R.,Wood, S., Guerry, A., Tallis, H., Ricketts, T. (2016): InVEST User's Guide. - The Natural Capital Project, Stanford University, University of Minnesota, The Nature Conservancy, and Word Wildlife Fund, Minneapolis, MN.

[23] Seto, K. C., Guneralp, B., Hutyra, L. R. (2012): Global forecasts of urban expansion to 2030 and direct impacts on biodiversity and carbon pool. - PNAS 109(40): 16083-16088. 


$$
-4597 \text { - }
$$

[24] Sun, X. Y., Jiang, Z., Liu, F., Zhang, D. Z. (2019): Monitoring spatio-temporal dynamics of habitat quality in Nansihu Lake basin, eastern China, from 1980 to 2015. - Ecological Indicator 102: 716-723.

[25] Tallis, H. T., Ricketts, T., Nelson, E., Ennaanay, D., Wolny, S., Olwero, N., Vigerstol, P. D., Mendoza, G., Aukema, J., Foster, J., Forrest, J., Cameron, D., Lonsdorf, E., Kennedy, C. (2010): InVEST 1.005 beta User's Guide. - The Natural Capital Project, Stanford.

[26] Tang, F., Fu, M., Wang, L., Zhang, P. (2020): Land-use change in Changli County, China: predicting its spatio-temporal evolution in habitat quality. - Ecological Indicators 117: 106719.

[27] Terrado, M., Sabater, S., Chaplin-Kramer, B., Mandle, L., Ziv, G., Acuna, V. (2016): Model development for the assessment of terrestrial and aquatic habitat quality in conservation planning. - Science of Total Environment 540: 63-70.

[28] Upadhaya, S., Dwivedi, P. (2019): Conversion of forestlands to blueberries: assessing implications for habitat quality in Alabaha river watershed in Southeastern Georgia, United States. - Land Use Policy 89: 104229.

[29] Wang, H., Xu, Y. Q., Liu, C., Huang, A., Lu, L. H., Zheng, W. R. (2019): Response of habitat quality to land use change based on geographical weighted regression. - Acta Scientiarum Naturalium Universitatis Pekinensis 55(3): 509-518.

[30] Wang, J. F., Wu, T. L., Li, Q., Wang, S. (2021): Quantifying the effect of environmental drivers on water conservation variation in the eastern Loess Plateau, China. - Ecological Indicators 125: 107493.

[31] Wu, J. S., Cao, Q. W., Shi, S. Q., Huang, X. L., Lu, Z. Q. (2015): Spatio-temporal variability of habitat quality in Beijing-Tianjin-Hebei Area based on land use change. Chinese Journal of Applied Ecology 26(11): 3457-3466.

[32] Zhang, Y. Z., Chen, R. S., Wang, Y. (2020): Tendency of land reclamation in coastal areas of Shanghai from 1998 to 2015. - Land Use Policy 91: 104370.

[33] Zhao, X. L., Zhang, Z. X., Wang, X., Zuo, L. J., Liu, B., Yi, L., Xu, J. Y., Wen, Q. K. (2014): Analysis of Chinese cultivated land's spatial-temporal changes and causes in recently 30 years. - Transactions of the Chinese Society of Agricultural Engineering 30(3): $1-11$. 